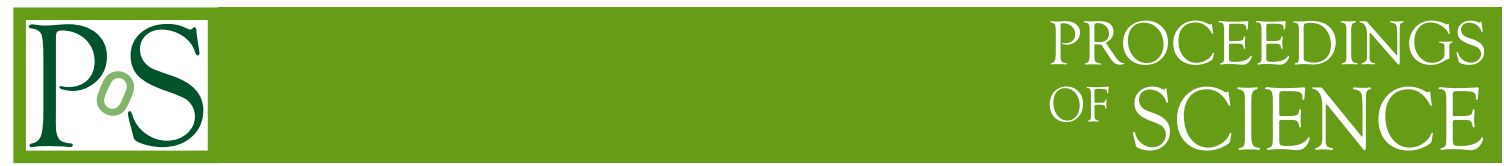

\title{
Physical predicions from lattice QCD
}

\author{
Christian Hoelbling* \\ Bergische Universität Wuppertal \\ E-mail: hch@physik.uni-wuppertal.de
}

Lattice QCD is a method for solving the nonperturbative dynamics of low energy QCD from first principles. Over the last few years, the field has matured considerably and reliable experimental predictions have been obtained in many areas. I will summarise the most important developments, give an overview of the currently attainable precision on key observables such as the hadron spectrum or the light quark masses and discuss some open challenges and future perspectives of the field.

Frontiers of Fundamental Physics 14 - FFP14,

15-18 July 2014

Aix Marseille University (AMU) Saint-Charles Campus, Marseille

${ }^{*}$ Speaker. 